\title{
Interrelationships of Pressure-Dependent Hole Fraction and Elongational Viscosity in Polymer Melts
}

\author{
Fatma Sahin-Dinc $\mathbb{D}^{1},{ }^{1}$ Ugur Yahsi, ${ }^{2}$ and Tomas Sedlacek ${ }^{3}$ \\ ${ }^{1}$ Karadeniz Technical University, Surmene Abdullah Kanca Vocational School, Machinery and Metal Technologies, \\ Machinery Department, 61530 Camburnu, Surmene, Trabzon, Turkey \\ ${ }^{2}$ Physics Department, Faculty of Arts and Sciences, Marmara University, Goztepe Campus, 34722 Kadikoy, Istanbul, Turkey \\ ${ }^{3}$ Department of Polymer Engineering, Faculty of Technology, Tomas Bata University in Zlin, Zlin, Czech Republic
}

Correspondence should be addressed to Fatma Sahin-Dinc; fatmadinc@ktu.edu.tr

Received 21 September 2018; Accepted 11 March 2019; Published 2 May 2019

Academic Editor: Volkan Filiz

Copyright (C) 2019 Fatma Sahin-Dinc et al. This is an open access article distributed under the Creative Commons Attribution License, which permits unrestricted use, distribution, and reproduction in any medium, provided the original work is properly cited.

\begin{abstract}
The elongational flow behavior of polyethylene, polypropylene, polystyrene, poly(methyl methacrylate), and polycarbonate, temperatures from 70 to $290^{\circ} \mathrm{C}$ and pressures up to $70 \mathrm{MPa}$, is examined with the Yahsi-Dinc-Tav (YDT) model and its particular case known as the Cross model. The viscosity data employed in the range of 3-405 $\mathrm{s}^{-1}$ elongational rates were acquired from the literature at ambient and elevated pressures. The predictions and the fitting results of the proposed YDT model with the same measurement data are compared with the Cross model. The average absolute deviations of the viscosities predicted by the YDT model range from $0.54 \%$ to $9.44 \%$ at ambient and $1.95 \%$ to $6.28 \%$ at high pressures. Additionally, the linear formulations derived from the YDT model are employed to relate the viscosity with temperature and hole fraction ("thermooccupancy" function) at zero level of elongational rate and constant elongational rate along with constant elongational stress. The effects of the four viscosity parameters (such as transmission and activation energy coefficients in these equations) on the elongational viscosity are analyzed in detail and some conclusions on the structural differences for the polymers are discussed.
\end{abstract}

\section{Introduction}

In rheology, flow is mostly a mixture of elongation and shear. Elongational dominated, shear dominated, in rare cases pure shear, or purely elongational flow is the nature of this phenomenon. Shear flow is typically used to characterize flow behavior since it is easily produced under laboratory conditions. Elongational viscosity which is known to generate strong stress responses also plays a crucial role for material characterization [1]. In some polymer melt processing only elongational properties can identify the polymeric materials having the same shear viscosity properties. In various manufacturing processes such as film blowing, fiber spinning, film casting, etc., elongational viscosity gives more accurate knowledge for polymer processibility as well [2]. The important attempt to reveal the underlying mechanisms behind these applications is modeling.
Several Newtonian and non-Newtonian models are present in the literature. However, none of the existing Newtonian equations is able to predict well the elongational flow. Unlike Newtonian equations, non-Newtonian models have a capability to more correctly represent nonlinear behavior of the polymer melts. Those most frequently applied to elongational viscosity is Sarkar-Gupta elongational viscosity model [3] and a few-parametric modified White-Metzner model (mWM) [4, 5]. Gupta et al. [3] fitted their shear viscosity values in terms of the Carreau model for the shearrate dependence. For elongational data, they introduced an equation which is a modificated form of the Carreau model to capture the strain hardening effect as it is in LDPE polymer $[1,3]$. Several authors, however, have attempted to develop composite models combining the shear and elongational viscosities data fitting applications $[4,5]$. Zatloukal employed the proposed modified Leonov, the eXtended Pom-Pom and 
the modification of White-Metzner equations for both shear and elongational flows of LDPE, mLLDPE, and PVB melts. He tested a new modification of the Leonov model with a proposed dissipation term and showed that the model results are very good fitting for all flow situations. In his study, while the XPP model accurately describes the elongational viscosity up to elongational rate about $10 \mathrm{~s}^{-1}$, at higher elongational rates, poor estimation is accomplished. In a similar manner, the elongational flow, in this paper, has been represented by the YDT (Yahsi-Dinc-Tav) model which has been successfully employed for the shear viscosity previously [6]. The model works successfully on both temperature- and pressure-dependent zero level of viscosities and critical stress parameter with a single fitting parameter. Analyses were carried out on HDPE, LDPE, LLDPE, PP, PC, PMMA, and PS melts at both low (3-50 $\mathrm{s}^{-1}$ ) and high (up to about $405 \mathrm{~s}^{-1}$ ) elongational rates in a wide range of $T$ and $P$ data.

The aim of the present paper as the first part of the work is to evaluate the suitability of the YDT equation in modeling of elongational flow. The fitting/predicting performance of the proposed YDT model is compared with the Cross-like model and better results were obtained for the former one. The viscosity data and the parameters determined from elongational viscosity were analyzed and correlated in terms of temperature and pressure by the YDT model.

In recent years there has been a considerable interest in free volume concept as a means to describe the mechanical behavior of polymer materials, particularly viscosity [6-14]. The term "free volume", which is expressed as an additional space needed for the thermal motion, is first introduced in Van der Waals' thesis [15]. More than a century ago, Batschinski [16] suggested the fact that the free volume is more decisive than the temperature for determining the viscosity of low-molecular weight liquids. This approach was further elaborated on polymers in the 1950 s by Doolittle $[17,18]$. In the late 1960s, Simha and Somcynsky derived an equation of state in their lattice-hole theory of liquids and introduced the hole fraction quantity as a measure of free volume [19]. Utracki presented an inverse relation of the logarithm of zero shear viscosity with hole fraction using a modified functional form of Doolittle's equation [7-10]. Sedlacek et al. [11] applied their modified Utracki's hole fraction model to linearize the zero shear and constant shearstress viscosities data. In recent articles, the YDT model by Dinc et al. $[6,13,14]$ offered a linear relation between shear-rate/shear-stress viscosities and temperature- and hole fraction-dependent thermooccupancy function for various polymers. Although to date several derived models have been developed to account for the relation of shear viscosity to the free volume [6-14], almost no studies have been carried out for the elongational viscosity.

In this work, as a second part, we focused on the effect of hole fraction on elongational flow. The dependence of elongational viscosity on hole fraction is analyzed in terms of linear equations derived from the YDT model at zero level of elongational rate, constant elongational rate, and constant elongational stress. From these derivations we also obtained the viscosity parameters, e.g., transmission coefficient and a measure of activation energy coefficient, and related them with constant elongational rate and constant elongational stress.

\section{Theories}

2.1. The Simha-Somcynsky (SS) Lattice-Hole Theory. The Simha and Somcynsky (SS) [19] lattice-hole theory developed in 1969 describes the pressure, volume, and temperature $(P V T)$ behavior of polymer melts and particularly introduces the term occupied site fraction, $y(P, T)$. The temperature- and pressure-dependent occupied lattice site fraction parameter, $y$, expressed as the ratio of the occupied sites on all of lattice sites, and the complementary hole fraction, $h$, are given by the following equation:

$$
h=1-y=\frac{N_{h}}{s N+N_{h}}
$$

where $s$ is the number of segments in a molecule and $N$ and $N_{h}$ are the number of molecules and holes, respectively.

The model includes $P, V, T$ variables in a scaled form $(\widetilde{P}$, $\widetilde{V}$, and $\widetilde{T})$, namely, $\widetilde{V}=V / V^{*}, \widetilde{T}=T / T^{*}$, and $\widetilde{P}=P / P^{*}$ with the characteristic scaling parameters, $V^{*}, T^{*}$, and $P^{*}$ :

$$
\begin{aligned}
V^{*} & =\frac{N_{A} v^{*}}{m_{0}}, \\
T^{*} & =\frac{q_{z} \varepsilon^{*}}{c k}, \\
\text { and } P^{*} & =\frac{q_{z} \varepsilon^{*}}{s v^{*}}
\end{aligned}
$$

where $N_{A}$ is the Avogadro's number, $m_{0}$ is the molar mass of a segment, and $k$ is the Boltzmann's constant. $v^{*}$ and $\varepsilon^{*}$ are, respectively, the characteristic molar volume and attractive energy parameter per segment. $q_{z}=s(z-2)+2$ is the number of the first neighbor intermolecular pairs of the $s$-mer (chain length of a polymer) in a lattice of the coordination number $z$. The theory incorporates a measure of chain flexibility expressed as the ratio of the external degree of freedom of the molecule, $3 c$, to the number of segments per molecule, $s$.

Using reduced variables $\widetilde{P}, \widetilde{V}, \widetilde{T}$ and hole fraction parameter, $h$, the SS provides two coupled equations:

$$
\begin{aligned}
& \frac{\widetilde{P} \widetilde{V}}{\widetilde{T}} \\
& =(1-Q)^{-1} \\
& \quad+\left(\frac{2 y}{\widetilde{T}}\right)(y \widetilde{V})^{-2}\left[1.011(y \widetilde{V})^{-2}-1.2045\right]
\end{aligned}
$$




$$
\begin{aligned}
\left(\frac{s}{3 c}\right) & {\left[\frac{(s-1)}{s}+y^{-1} \ln (1-y)\right] } \\
= & \frac{(Q-1 / 3)}{(1-Q)} \\
& +\left(\frac{y}{6 \widetilde{T}}\right)(y \widetilde{V})^{-2}\left[2.409-3.033(y \widetilde{V})^{-2}\right]
\end{aligned}
$$

where $Q=2^{-1 / 6} y(y \widetilde{V})^{-1 / 3}$.

The characteristic scaling parameters, $V^{*}, T^{*}, P^{*}$ as well as temperature- and pressure-dependent hole fraction $h(P, T)$ parameter can be determined by superimposing experimental $P V T$ data on the theoretical $\widetilde{P}-\widetilde{V}-\widetilde{T}$ surface with the use of (3) and (4). The model is advantageous in that it provides the complementary hole fraction, $h$, which has been correlated with transport properties and explains the mechanical behavior of polymers such as viscosity $[6,10]$, ionic conductivity [20], etc.

2.2. The Yahsi-Dinc-Tav (YDT) Model. We have shown previously that the Yahsi-Dinc-Tav (YDT) non-Newtonian viscosity model fitted well the shear viscosity data particularly at high pressure [6]. For the bulk structured polymers like PMMA, PC, and PS of which the viscosity values are high comparing the remaining polymers, the outcomes were found reasonable as well. This result led us to test the YDT model for the high viscosity values as in elongational flow. Hence, one of the aims in the present study is primarily not to observe the results, but to prove our foresight about the suitability of the YDT model for predicting elongational viscosity.

Here, elongational behavior of the same polymers is going to be modeled with the same equation which mathematically relates Newtonian viscosity (zero level of elongational viscosity), $\eta_{0}$, and elongational rate, as $\dot{\varepsilon}$ :

$$
\begin{aligned}
& \eta_{E} \\
& =\frac{\eta_{0}}{1+\left(\theta_{1} / \Gamma(q+1)\right)\left(\eta_{0} \dot{\varepsilon} / \tau\right)^{q}+\left(\theta_{2} / \Gamma(2 q+1)\right)\left(\eta_{0} \dot{\varepsilon} / \tau\right)^{2 q}}
\end{aligned}
$$

Neglecting $2 q$ power term in the denominator of (5), we obtain the Cross-like model $[6,13,14]$;

$$
\eta_{E}=\frac{\eta_{0}}{1+\left(\theta_{1} / \Gamma(q+1)\right)\left(\eta_{0} \dot{\varepsilon} / \tau\right)^{q}}
$$

where the nonlinearity parameter $q$ is the order of the fractional derivative of the exponential function in the Eyring's strain rate [6] and $\Gamma(q)$ denotes the gamma function. $\theta_{n}=$ $(2 / \pi) \sum_{i} \cos ^{n q+2} \theta_{i},(n=1,2)$ is a measure of the characteristic ratio, involved the angle, $\theta_{i}$, between the deformation stress and the displacement vector of the segment jumping into the neighboring $i$ th lattice position. $n$ denotes the sequential order of the fractional derivative and the fractional integral in the fractional Taylor series expansion of the first exponential product in the Eyring's rate of strain in (3) of [6]. $\tau=$ $4 R T / \sqrt{6} v$ is the critical stress parameter in which $R$ and $v$ are gas constant and the molar volume of a segment, respectively.
2.3. The Viscosity Model in Terms of Thermooccupancy Function. A linear relation between the viscosities and thermooccupancy function, $Y_{h}$, is $[6,12-14]$

$$
\ln \eta_{E}=\ln \eta_{E}^{*}+\alpha Y_{h} ; \quad Y_{h}=\frac{1-h}{h} \frac{1}{T}
$$

where $\eta_{E}$ is zero level of elongational viscosity (Newtonian viscosity) $\left(\eta_{E}=\eta_{0}\right)$, constant elongational rate viscosity $\left(\eta_{E}=\eta_{\dot{\varepsilon}}\right)$, or constant elongational stress viscosity $\left(\eta_{E}=\right.$ $\left.\eta_{\sigma}\right) . Y_{h}$ depends on the structural occupancy-nonoccupancy ratio $((1-h) / h)$ and inversely related to the temperature. The viscosity-intercept $\ln \eta_{E}^{*}$ (for the zero level of elongational rate, constant elongational rate, and constant elongational stress; $\ln \eta_{E}=\ln \eta_{E}^{*}=\ln \eta_{\dot{\varepsilon}_{0}}^{*}, \ln \eta_{E}=\ln \eta_{E}^{*}=\ln \eta_{\dot{\varepsilon}}^{*}$, or $\ln \eta_{E}=\ln \eta_{E}^{*}=\ln \eta_{\sigma}^{*}$, respectively) represents the extrapolated viscosity values at zero $Y_{h}$ (i.e., when the system is dominated by large hole fraction at high temperature and low pressure). The slope $\alpha$ refers to a measure of activation energy at zero level of elongational rate $\left(\alpha=\alpha_{\dot{\varepsilon}_{0}}\right)$, constant elongational rate $\left(\alpha=\alpha_{\dot{\varepsilon}}\right)$, or constant elongational stress $\left(\alpha=\alpha_{\sigma}\right)$. The parameters $\eta^{*}$ and $\alpha$ are given by

$$
\begin{aligned}
\eta_{E}^{*} & =\frac{\sqrt{2} N_{A} h_{p}}{\pi \kappa v} \\
\text { and } \alpha & =a^{\prime} \frac{q_{z} \Phi}{2 k}
\end{aligned}
$$

where $h_{p}$ and $k$ are Planck and Boltzmann's constants, respectively. $\kappa$ is the transmission coefficient which is a measure of possibility of a molecule to move from one site to a vacant site. $a^{\prime}$ is the proportionality constant of activation energy and $\Phi$ (assumed to be constant [6]) is the interaction potential energy between a pair of segments of a polymer.

\section{Calculations and Discussion}

3.1. The SS Theory Parameters. We analyzed the PVT behavior of some commercially available polymers of highdensity polyethylene (HDPE), low-density polyethylene (LDPE60 and LDPE65), linear low-density polyethylene (LLDPE), polypropylene (PP), polystyrene (PS), poly(methyl methacrylate) (PMMA), and polycarbonate (PC). Their experimental PVT data reported by Sedlacek et al. [11] were accomplished on a fully automated PVT instrument (pvT 100 SWO, Germany) in the temperature range $70-290^{\circ} \mathrm{C}$ and the pressure range 15-70 $\mathrm{MPa}$ with the increments of $5 \mathrm{MPa}$.

In the present paper we used the values of the characteristic scaling parameters $P^{*}, V^{*}, T^{*}$ and the structural flexibility parameter $3 c / s$ with $3 c=s+3$ computed from the experimental data by the use of the coupled equations (3) and (4) from our previous work [6] in which LDPE 65 was excluded (LDPE refers to LDPE 60 in ref [6]). For the corresponding polymer the scaling parameters and the segment number are computed as $V^{*}=1.1723 \mathrm{~cm}^{3} / \mathrm{g}$, $T^{*}=10506.2 \mathrm{~K}, P^{*}=609 \mathrm{MPa},\langle-\Phi / k\rangle=503.02 \mathrm{~K}$, and $s=2007$ with the same method explained before [6]. These parameters are used for the determination of hole fraction at each temperature and pressure and later used in viscosity calculations. 


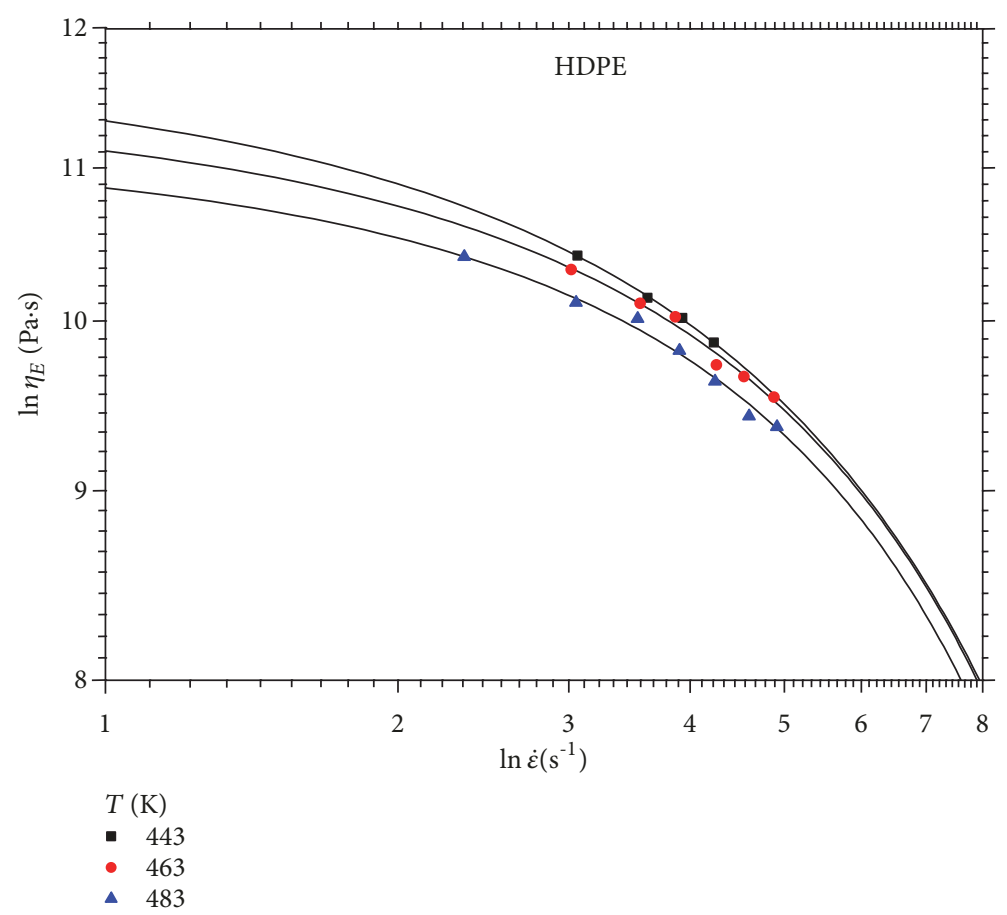

FIGURE 1: Logarithm of viscosity versus logarithm of elongational rate of HDPE at atmospheric pressure and various temperatures. Solid lines represent data fitting through each data set [4] by (5).

3.2. The Elongational Viscosity Data Fit. The experimental rheological data for the polymers studied were reported by Sedlacek et al. [4] for three tested temperatures and six pressures $(0.1,10,20,35,50$, and $70 \mathrm{MPa})$. The elongational rate dependent viscosity was measured in a modified capillary rheometer (Göttfert 2001, Germany). A back-pressure device in order to raise the pressure and two capillaries with lengthto-diameter ratio of 0.12 and 20 were used. The measurements were complemented by employing the Cogswell analysis together with pressure-dependent entrance pressure drops. The effect of viscous heating was omitted during the measurements because the magnitudes of the Nahme number were found to be less than 1. A closer description of the experimental technique and instrumentation can be found in the preceding article [4].

Now, these sets of data are fitted for each polymer using (5) and (6) with the double and single nonvanishing term(s) in the denominator, respectively, to obtain the zero level of elongational viscosities, $\eta_{0}$, and the stress parameters, $\tau$, along with the fraction $q$ as a nonlinearity parameter in the power of elongational rate. Our motivation is to obtain $\eta_{0}$ and $\tau$ for each temperature and pressure data set while $q$ is a sole parameter for each polymer. To obtain $\eta_{0}$ and $\tau$, we employ nonlinear fit scheme assuming that $q$ is taken as a disposable quantity. The best $q$ value is adjusted with the minimum average absolute deviation in viscosity defined as (relative mean absolute percentage error)

$$
\Delta \eta_{E}(\%)=\frac{100}{N} \sum_{i}\left|1-\frac{\eta_{i}^{\text {calc }}}{\eta_{i}^{\exp }}\right|
$$

The viscosity data are given in the range of 3 to $405 \mathrm{~s}^{-1}$ elongational rate. These datasets can be used to obtain $\eta_{0}$ and $\tau$ as well as $q$ parameter explained above scheme. For high pressures, we obtain all $\eta_{0}$ and $\tau$ by fixing $q$ value at ambient pressure. The $\eta_{0}$ and $\tau$ values at ambient pressure are presented in Table 1 alongside with mean percentage relative errors in viscosity, $\Delta \eta_{E}(\%)$, and correlation coefficient squared, $R^{2}$. The latter, also called the coefficient of determination, is a measure of how much of the variance in experimental value of $\eta_{E}$ is explained by the model. The percentages relative errors are computed by using (9). The values in parentheses are calculated using (6) while the rest with (5). To reduce figure crowding, we demonstrated HDPE at Figures 1, 2, and 4 as well as PMMA at Figure 3. Logarithmic elongational viscosity versus logarithmic elongational rate of HDPE is plotted at various temperatures at ambient pressure in Figure 1 and for various pressures only at $210{ }^{\circ} \mathrm{C}$ in Figure 2. Similar plots are obtained for the other polymers. The solid line is drawn by (5) with the best fit parameters. These parameters at ambient pressure are collected in Table 1 with $\Delta \eta_{E}(\%)$ and $R^{2}$. In a similar manner, the values of the parameters in (6) are also tabulated in parenthesis. When we compare the result we can say that $\Delta \eta_{E}(\%)$ does not differ according to both models for HDPE. Only for PP the Cross-like model has a better capability to describe the viscosity values, while for the remaining polymers the values are estimated better with the YDT model. Specifically, from Table 1, it can be observed that, with the YDT model, good estimation is achieved for the linear or nearly linear polymers (HDPE; LLDPE) and PP with simple structure. The fitting is reasonable for linear long chain branched (LDPEs) and large 
TABLE 1: Rheological parameters computed from (5) and (6) in parenthesis in terms of three different temperatures at ambient pressure.

\begin{tabular}{|c|c|c|c|c|c|c|c|c|}
\hline Polymer & $T\left({ }^{\circ} \mathrm{C}\right)$ & $q$ & $\theta_{1}$ & $\Gamma(q+1)$ & $\eta_{0}($ Pa.s $)$ & $\tau(\mathrm{Pa})$ & $\Delta \eta_{E}(\%)$ & $R^{2}$ \\
\hline \multirow{5}{*}{ LDPE60 } & 150 & \multirow{5}{*}{$\begin{array}{c}0.655 \\
(0.507)\end{array}$} & \multirow{5}{*}{$\begin{array}{c}0.238 \\
(0.447)\end{array}$} & \multirow{5}{*}{$\begin{array}{c}0.901 \\
(0.886)\end{array}$} & $\begin{array}{c}17535.7 \\
(25744.2)\end{array}$ & $\begin{array}{l}9.9122 \times 10^{7} \\
\left(1.999 \times 10^{6}\right)\end{array}$ & $\begin{array}{c}2.82 \\
(6.14)\end{array}$ & $\begin{array}{c}0.99898 \\
(0.99234)\end{array}$ \\
\hline & 170 & & & & 17291.2 & 767495 & 7.24 & 0.99429 \\
\hline & $1 / 0$ & & & & $(24243.4)$ & $(244722)$ & $(4.49)$ & $(0.99793)$ \\
\hline & 100 & & & & 8002.41 & $1.58501 \times 10^{6}$ & 1.89 & 0.99977 \\
\hline & 190 & & & & (9094.53) & $\left(1.3827 \times 10^{6}\right)$ & $(1.95)$ & $(0.99961)$ \\
\hline \multirow{5}{*}{ LDPE65 } & 150 & \multirow{5}{*}{$\begin{array}{c}0.660 \\
(0.820)\end{array}$} & \multirow{5}{*}{$\begin{array}{c}0.231 \\
(0.068)\end{array}$} & \multirow{5}{*}{$\begin{array}{c}0.902 \\
(0.937)\end{array}$} & $\begin{array}{l}50800.3 \\
(51657.7)\end{array}$ & $\begin{array}{c}2.03715 \times 10^{6} \\
(221494)\end{array}$ & $\begin{array}{c}8.46 \\
(9.38)\end{array}$ & $\begin{array}{c}0.99155 \\
(0.99002)\end{array}$ \\
\hline & & & & & 24061.8 & $1.04557 \times 10^{9}$ & 2.83 & 0.99887 \\
\hline & 170 & & & & (24089.5) & $\left(3.5902 \times 10^{7}\right)$ & $(2.83)$ & $(0.99893)$ \\
\hline & & & & & 16430 & $2.27597 \times 10^{7}$ & 3.45 & 0.99860 \\
\hline & 190 & & & & $(16364)$ & $\left(2.8256 \times 10^{6}\right)$ & $(3.44)$ & $(0.99860)$ \\
\hline \multirow{6}{*}{ LLDPE } & 150 & \multirow{6}{*}{$\begin{array}{c}0.547 \\
(0.649)\end{array}$} & \multirow{6}{*}{$\begin{array}{c}0.387 \\
(0.245)\end{array}$} & \multirow{6}{*}{$\begin{array}{c}0.889 \\
(0.900)\end{array}$} & 29500.4 & 208213 & 2.44 & 0.99903 \\
\hline & 100 & & & & $(27429.7)$ & (130950) & $(2.71)$ & $(0.99893)$ \\
\hline & & & & & 15524.9 & 306655 & 0.68 & 0.99994 \\
\hline & 170 & & & & $(14189.6)$ & $(199260)$ & $(0.68)$ & $(0.99994)$ \\
\hline & 190 & & & & 14798.2 & 208924 & 4.94 & 0.99748 \\
\hline & 190 & & & & $(13531.6)$ & (136025) & $(5.00)$ & $(0.99755)$ \\
\hline \multirow{6}{*}{ HDPE } & 170 & \multirow{6}{*}{$\begin{array}{c}0.499 \\
(0.494)\end{array}$} & \multirow{6}{*}{$\begin{array}{c}0.459 \\
(0.467)\end{array}$} & \multirow{6}{*}{$\begin{array}{c}0.886 \\
(0.886)\end{array}$} & 396457 & 18863.3 & 0.54 & 0.99996 \\
\hline & $1 / 0$ & & & & $(464905)$ & (15151.1) & $(0.53)$ & (0.99996) \\
\hline & 190 & & & & 174351 & 43466.5 & 2.70 & 0.99895 \\
\hline & 190 & & & & (187253) & (38795.4) & $(2.71)$ & $(0.99894)$ \\
\hline & 210 & & & & 115812 & 51574.5 & 3.01 & 0.99887 \\
\hline & 210 & & & & (120758) & $(47922.2)$ & (3.01) & $(0.99886)$ \\
\hline \multirow{6}{*}{ PMMA } & 230 & \multirow{6}{*}{$\begin{array}{c}0.523 \\
(0.630)\end{array}$} & \multirow{6}{*}{$\begin{array}{c}0.423 \\
(0.270)\end{array}$} & \multirow{6}{*}{$\begin{array}{c}0.887 \\
(0.897)\end{array}$} & 243538 & 127536 & 3.27 & 0.99886 \\
\hline & 230 & & & & $(281487)$ & (114841) & $(7.17)$ & $(0.98958)$ \\
\hline & 240 & & & & 239972 & 56457.2 & 6.06 & 0.99688 \\
\hline & 240 & & & & $(230746)$ & $(51143.9)$ & $(7.13)$ & $(0.99513)$ \\
\hline & 250 & & & & 177553 & 34318.5 & 5.28 & 0.99750 \\
\hline & 250 & & & & $(157033)$ & $(36123.8)$ & $(4.95)$ & $(0.99780)$ \\
\hline \multirow{6}{*}{ PC } & & & & & 3031.34 & 553155 & 6.51 & 0.99359 \\
\hline & 280 & & & & $(4591.78)$ & (39800.3) & (7.91) & $(0.99156)$ \\
\hline & & & 0.059 & 0.940 & 3535.29 & 698105 & 5.06 & 0.99687 \\
\hline & 290 & $(0.757)$ & $(0.004)$ & $(0.982)$ & $(4167.8)$ & (95812.8) & $(7.13)$ & $(0.99468)$ \\
\hline & 200 & & & & 3886.5 & 480617 & 7.34 & 0.99496 \\
\hline & 300 & & & & (7982.78) & $(26169.9)$ & $(5.20)$ & $(0.99748)$ \\
\hline & & & & & 146262 & 10432.9 & 3.08 & 0.99872 \\
\hline & 190 & & & & (52099.9) & $(17176.4)$ & $(2.93)$ & $(0.99890)$ \\
\hline PP & 210 & 0.258 & 0.462 & 0.8861 & 115212 & 10760.2 & 3.83 & 0.99822 \\
\hline$P P$ & 210 & $(0.486)$ & $(0.479)$ & $(0.886)$ & $(50774)$ & (13328.4) & $(3.88)$ & $(0.99815)$ \\
\hline & 230 & & & & 108176 & 6192.31 & 0.55 & 0.99997 \\
\hline & 230 & & & & $(44800.8)$ & $(8330.87)$ & $(0.56)$ & $(0.99997)$ \\
\hline & & & & & 101383 & 361180 & 3.96 & 0.99837 \\
\hline & 190 & & & & $(140943)$ & (122334) & $(4.05)$ & $(0.99844)$ \\
\hline PS & 210 & 0.580 & 0.339 & 0.891 & 17697.6 & 491802 & 9.44 & 0.99014 \\
\hline PS & 210 & $(0.552)$ & $(0.380)$ & $(0.889)$ & (19396.4) & (306492) & $(8.91)$ & $(0.99107)$ \\
\hline & 230 & & & & 15978.4 & 172216 & 4.98 & 0.99671 \\
\hline & 250 & & & & (13049.7) & (184296) & $(9.76)$ & $(0.99006)$ \\
\hline
\end{tabular}

group attached polymers like PMMA, PC, and PS. The mean percentage errors in viscosity for all the polymers considered are less than $6.30 \%$ at ambient and $6.28 \%$ at higher pressures. However, using (6), the mean percentage errors are less than $7.58 \%$ at ambient pressure and $6.59 \%$ at higher pressures. Overall, $\Delta \eta_{E}(\%)$ according to (5) decreases almost $0.5 \%$ at ambient and $0.8 \%$ at high pressures comparing to (6). Hence (5) better fits the viscosity data than (6) both at ambient and at high pressures. In the previous findings, the observed nonNewtonian behavior is better described by the Cross model only at ambient pressure (see [6]).

Moreover, in Figure 3, the zero level of elongational rate viscosity $\left(\eta_{0}\right)$ and the stress parameter $(\tau)$ are plotted as a function of pressure at various temperatures for PMMA. For the zero level of elongational rate viscosity the graph includes the best fit line. The zero elongational viscosity 


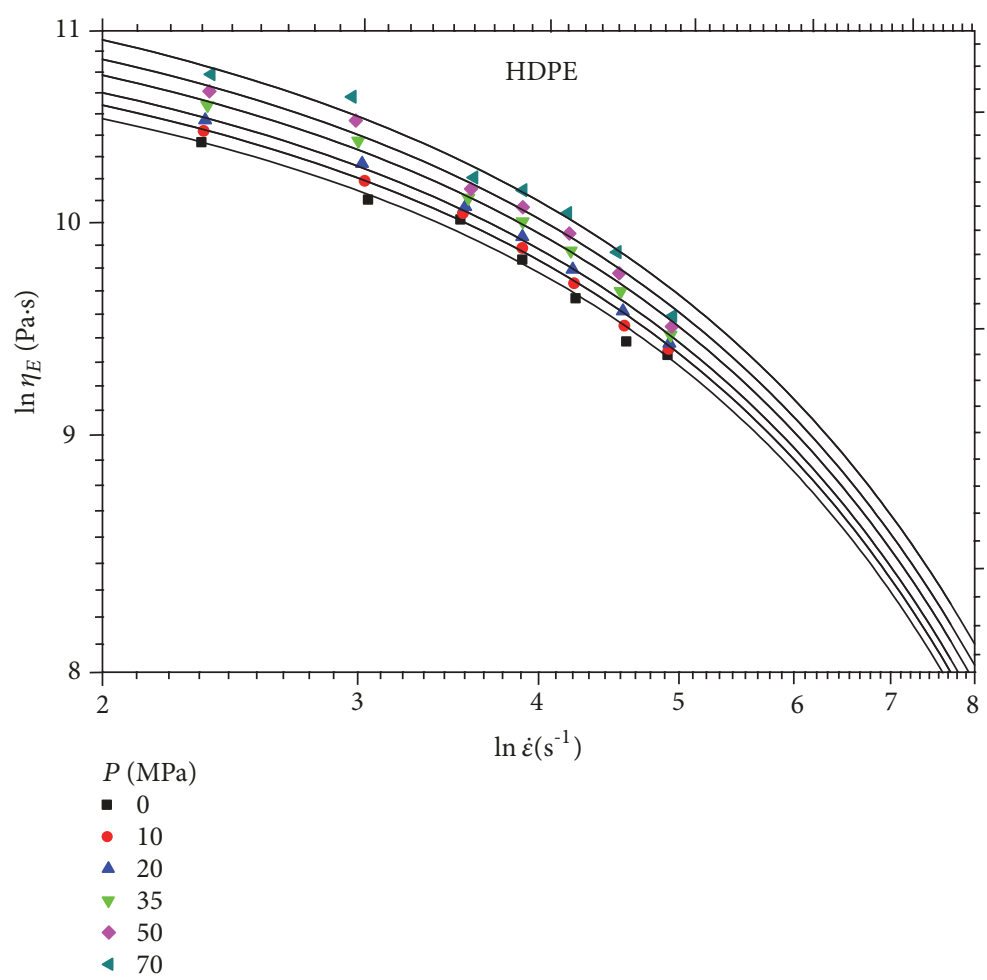

FIGURE 2: Logarithm of viscosity versus logarithm of elongational rate of $\mathrm{HDPE}$ at $210{ }^{\circ} \mathrm{C}$ and various pressures. Solid lines represent data fitting through each data set [4] by (5).

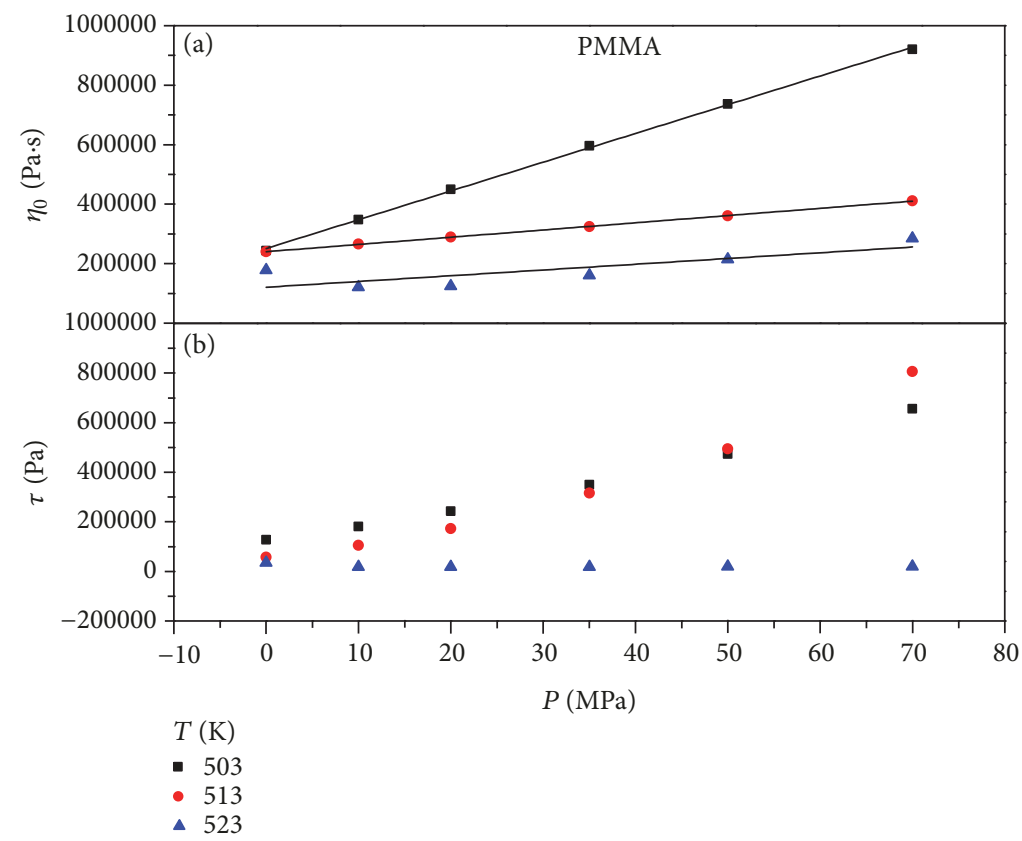

FIGURE 3: Zero level of elongational viscosities, $\eta_{0}$, and critical stress parameter, $\tau$, computed by (5), with respect to pressures for PMMA at different temperatures. Solid lines represent linear fitting through the data [4].

increases linearly with increasing pressure, but the stress parameter, depending on pressure in terms of $T / v$, increases but not consistently in temperature. On the other hand, the zero elongational viscosity decreases steadily with increasing temperature, but we may not draw an exact temperature dependency on the stress parameter. The behavior of $\eta_{0}$ and $\tau$ with respect to pressure at elongational flow is similar to the one at shear flow (see [6]). 
TABLE 2: Values of parameters of (7) evaluated for $\eta_{0}$ at zero level of elongational rate according to (5) and (6) in brackets.

\begin{tabular}{|c|c|c|c|c|c|c|c|}
\hline Polymer & $\ln \eta_{\dot{\varepsilon}_{0}}^{*}$ & $q_{z}$ & $\alpha_{\dot{\varepsilon}_{0}}$ & $a_{\dot{\varepsilon}_{0}}^{\prime} \times\left(10^{4}\right)$ & $\kappa$ & $\Delta \eta_{E}(\%)$ & $R^{2}$ \\
\hline LDPE60 & $\begin{array}{c}7.60 \\
(8.02)\end{array}$ & 20372 & $\begin{array}{c}65.35 \\
(57.89)\end{array}$ & $\begin{array}{c}0.13 \\
(0.11)\end{array}$ & $\begin{array}{c}0.0020 \\
(0.0013)\end{array}$ & $\begin{array}{c}1.41 \\
(1.62)\end{array}$ & $\begin{array}{c}0.99972 \\
(0.99965)\end{array}$ \\
\hline LDPE65 & $\begin{array}{c}7.53 \\
(7.37)\end{array}$ & 20072 & $\begin{array}{c}87.01 \\
(92.81)\end{array}$ & $\begin{array}{c}0.17 \\
(0.18)\end{array}$ & $\begin{array}{c}0.0021 \\
(0.0024)\end{array}$ & $\begin{array}{c}1.35 \\
(1.58)\end{array}$ & $\begin{array}{c}0.99966 \\
(0.99953)\end{array}$ \\
\hline LLDPE & $\begin{array}{c}8.25 \\
(8.39) \\
\end{array}$ & 14672 & $\begin{array}{c}55.33 \\
(48.67) \\
\end{array}$ & $\begin{array}{c}0.15 \\
(0.13) \\
\end{array}$ & $\begin{array}{c}0.0010 \\
(0.00089) \\
\end{array}$ & $\begin{array}{c}1.45 \\
(1.47) \\
\end{array}$ & $\begin{array}{c}0.99970 \\
(0.99969) \\
\end{array}$ \\
\hline HDPE & $\begin{array}{c}9.85 \\
(9.66) \\
\end{array}$ & 28772 & $\begin{array}{c}126.93 \\
(141.26)\end{array}$ & $\begin{array}{c}0.21 \\
(0.23) \\
\end{array}$ & $\begin{array}{c}0.00024 \\
(0.00029)\end{array}$ & $\begin{array}{c}1.81 \\
(1.08) \\
\end{array}$ & $\begin{array}{c}0.99980 \\
(0.99975)\end{array}$ \\
\hline PMMA & $\begin{array}{c}6.72 \\
(6.82) \\
\end{array}$ & 29072 & $\begin{array}{c}358.19 \\
(334.90)\end{array}$ & $\begin{array}{c}0.53 \\
(0.50) \\
\end{array}$ & $\begin{array}{c}0.0068 \\
(0.0062) \\
\end{array}$ & $\begin{array}{c}1.81 \\
(2.21) \\
\end{array}$ & $\begin{array}{c}0.99961 \\
(0.99935)\end{array}$ \\
\hline PC & $\begin{array}{c}3.99 \\
(4.52) \\
\end{array}$ & 4022 & $\begin{array}{c}560.31 \\
(326.49) \\
\end{array}$ & $\begin{array}{c}3.83 \\
(3.48) \\
\end{array}$ & $\begin{array}{c}0.0926 \\
(0.0547) \\
\end{array}$ & $\begin{array}{c}1.62 \\
(1.96)\end{array}$ & $\begin{array}{c}0.99961 \\
(0.99942)\end{array}$ \\
\hline PP & $\begin{array}{c}8.87 \\
(8.39) \\
\end{array}$ & 49112 & $\begin{array}{c}95.51 \\
(121.62)\end{array}$ & $\begin{array}{l}0.090 \\
(0.12)\end{array}$ & $\begin{array}{c}0.00054 \\
(0.00088)\end{array}$ & $\begin{array}{c}2.08 \\
(2.27) \\
\end{array}$ & $\begin{array}{c}0.99910 \\
(0.99922)\end{array}$ \\
\hline PS & $\begin{array}{c}2.68 \\
(2.35)\end{array}$ & 66572 & $\begin{array}{c}390.21 \\
(411.54)\end{array}$ & $\begin{array}{l}-0.25 \\
(-0.26)\end{array}$ & $\begin{array}{c}0.306 \\
(0.426)\end{array}$ & $\begin{array}{c}3.32 \\
(3.54)\end{array}$ & $\begin{array}{c}0.99854 \\
(0.99824)\end{array}$ \\
\hline
\end{tabular}

\subsection{Elongational Viscosity-Hole Fraction Dependence under} Constant Elongational Rate and Stress. The relationship between hole fraction and viscosities at zero and constant elongational rates and constant elongational stress given in (7) is tested. The zero level of elongational viscosity values is estimated from the experimental data by using both the YDT and the Cross-like models (see (5) and (6), respectively), for the studied polymers. With regard to the constant elongational viscosity, the data are evaluated properly through the polynomial function fit within the measured elongational rate and pressure ranges as follows: the viscosity data are fitted with respect to elongational rate by a third-order (for LDPE 65, LLDPE, PMMA, PP, and PS), second-order (for LDPE 60 and HDPE), and first-order polynomial equations (for PC). The interpolating $\eta_{\dot{\varepsilon}}$ values are calculated at constant elongational rate values, $\dot{\varepsilon}_{c}$, in Table 3 , at the three temperatures and various pressure levels discussed earlier in explaining the computation of elongational viscosity. Similarly, the constant elongational stress viscosities, $\eta_{\sigma}$, are calculated at certain constant elongational stress, $\sigma_{c}$, in Table 4 , at the same temperature and pressure levels. The obtained zero elongational, constant elongational rates and constant elongational stress viscosity data are fitted to (7), in which the hole fraction is calculated by the SS theory. The regression parameters derived from the equation, $\ln \eta_{\dot{\varepsilon}_{0}}^{*}, \ln \eta_{\dot{\varepsilon}}^{*}, \ln \eta_{\sigma}^{*}$ and $\alpha_{\dot{\varepsilon}_{0}}, \alpha_{\dot{\varepsilon}}, \alpha_{\sigma}$, are presented in Tables $2-4$ with the correlation coefficient and $\Delta \eta_{E}(\%)$ obtained from (9) for the polymers. Using the best fit parameters, the values of $\Delta \eta_{E}(\%)$ in zero elongational, constant elongational rates and constant elongational stress are ranging from $1.35 \%$ to $3.32 \%, 0.23 \%$ to $2.44 \%$ and $0.47 \%$ to $4.97 \%$, respectively. $\Delta \eta_{E}(\%)$ in average for zero elongational viscosity is found to be $1.87 \%$ via the YDT model with a better accuracy compared to the Cross model of which deviation is $1.97 \%$ according to the results listed in Table 2.

In Figure 4, the logarithms of zero elongational rate, constant elongational rate, and constant elongational stress viscosities are plotted with respect to $Y_{h}$ for HDPE in which the solid lines are drawn through the data with the best fit line. A good linearization, for all conditions above, is achieved for the three tested temperatures and for all given pressures. In the figure, the pressure increases from left (the lower part) to right (the higher part) along each line and at each temperature. As the hole fraction decreases, $Y_{h}$ increases for each temperature, so the viscosity increases. Each line slope gives the value of $\alpha$, in Kelvin unit, evident from (7) and (8). As expected, in Figure 4, the logarithm of viscosities, $\ln \eta_{E}$, decreases as the elongational rate and elongational stress increase for HDPE.

For comparison, the special cases of Figure 4 at 90 $\mathrm{s}^{-1}$ elongational rate and certain elongation stresses are delineated in Figures 5 and 6, respectively. Figure 5 shows a plot of $\ln \eta_{E}$ versus $Y_{h}$ at $90 \mathrm{~s}^{-1}$ and Figure 6 displays $\ln \eta_{E}$ versus $Y_{h}$ graph at certain elongational stresses (the elongational stresses used in Figure 6 indicated with italic font in Table 4). Overall, we conclude that, for the elongational rate and all elongational stress values, polymers with simpler structures, PEs and PP, show slowly varying viscous behavior with respect to the thermooccupancy function, $Y_{h}$, so their slopes are smaller than PMMA, PC, and PS. PMMA has the highest thermooccupancy functional dependency, which seems to result from two pendant groups $\left(\mathrm{CH}_{3}\right.$ and $\left.\mathrm{CO}_{2} \mathrm{CH}_{3}\right)$ on each of its monomer units. Accordingly, the measure of the activation energy coefficient, $\alpha$, positively correlates with activation energy; thus this indicates that PMMA, PC, and PS polymers require more activation energy, $E_{a}$, than polyolefins (PP and PEs). The differences in $\alpha_{\dot{\varepsilon}}$ and $\alpha_{\sigma}$ values from Tables 3 and 4 can relatively be attributed to the large benzene ring on the former structures and related to the equilibrium shape of the chain [21]. Figures 7 and 8 graphs $\alpha_{\dot{\varepsilon}}$ and $\alpha_{\sigma}$ with respect to elongational rate and stress and provide a clearer picture to this pattern.

The computed $\alpha_{\dot{\varepsilon}}$ and $\alpha_{\sigma}$ parameters are analyzed at various constant elongational rate and constant elongational stress values including those in non-Newtonian region. At 


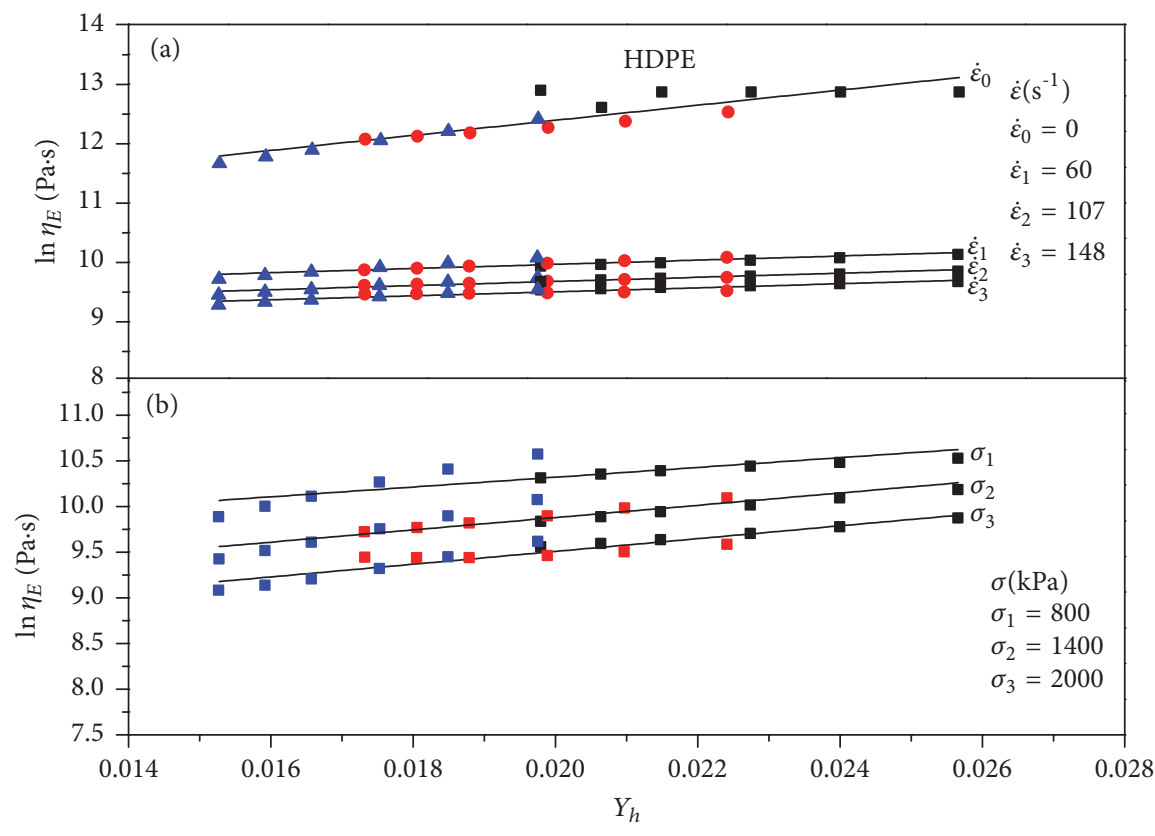

$T(\mathrm{~K})$

- 443

- 463

- 483

FIgURE 4: The logarithm of viscosities versus thermooccupancy function $Y_{h}$ for HDPE (a) at the zero level of elongational and the constant elongational rates $\left(\dot{\varepsilon}_{c}=60,107\right.$, and $\left.148 \mathrm{~s}^{-1}\right)$, and (b) constant elongational stresses $\left(\sigma_{c}=800,1400\right.$, and $\left.2000 \mathrm{kPa}\right)$. Solid lines represent linear fitting through each data set by (7) and (8) with the parameters tabulated in Tables 2-4.

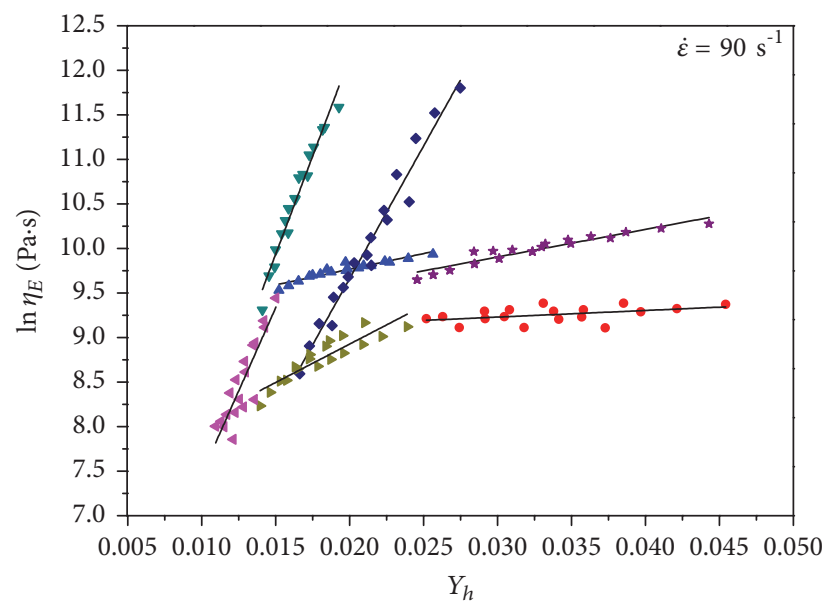

* LDPE65

$\triangle$ HDPE

- LLDPE

- PMMA

- $\mathrm{PP}$

$\triangleleft \mathrm{PC}$

- PS

Figure 5: The logarithm of viscosity at $90 \mathrm{~s}^{-1}$ elongational rate versus $Y_{h}$. The solid line is the best fit line through each data set by (7) and (8) with the parameters tabulated in Table 3. 
TABLE 3: Values of parameters of (7) evaluated for $\eta_{E}$ at different elongational rates.

\begin{tabular}{|c|c|c|c|c|c|c|c|}
\hline Polymer & $\dot{\varepsilon}_{c}\left(\mathrm{~s}^{-1}\right)$ & $\ln \eta_{\dot{\varepsilon}}^{*}$ & $\alpha_{\dot{\varepsilon}}$ & $a_{\dot{\varepsilon}}^{\prime} \times\left(10^{4}\right)$ & $\kappa$ & $\Delta \eta_{E}(\%)$ & $R^{2}$ \\
\hline \multirow{3}{*}{ LDPE60 } & 60 & 7.14 & 72.63 & 0.142 & 0.0031 & 1.18 & 0.99981 \\
\hline & 90 & 7.08 & 72.01 & 0.141 & 0.0033 & 1.32 & 0.99975 \\
\hline & 120 & 7.12 & 68.95 & 0.135 & 0.0032 & 1.33 & 0.99977 \\
\hline \multirow{4}{*}{ LDPE65 } & 30 & 8.05 & 67.69 & 0.134 & 0.0012 & 1.23 & 0.99977 \\
\hline & 60 & 8.61 & 46.81 & 0.0927 & 0.00070 & 1.07 & 0.99986 \\
\hline & 90 & 8.97 & 31.02 & 0.0615 & 0.00049 & 0.43 & 0.99997 \\
\hline & 107 & 9.10 & 24.13 & 0.048 & 0.00043 & 0.55 & 0.99995 \\
\hline \multirow{3}{*}{ LLDPE } & 60 & 9.13 & 8.47 & 0.0227 & 0.00042 & 0.64 & 0.99993 \\
\hline & 90 & 9.01 & 7.44 & 0.0199 & 0.00048 & 0.64 & 0.99993 \\
\hline & 120 & 8.89 & 7.06 & 0.0189 & 0.00054 & 0.60 & 0.99994 \\
\hline \multirow{5}{*}{ HDPE } & 60 & 9.26 & 35.23 & 0.0575 & 0.00043 & 0.34 & 0.99998 \\
\hline & 90 & 9.06 & 35.15 & 0.0574 & 0.00053 & 0.25 & 0.99999 \\
\hline & 107 & 8.98 & 34.86 & 0.0569 & 0.00057 & 0.23 & 0.99999 \\
\hline & 120 & 8.92 & 34.58 & 0.0564 & 0.00060 & 0.24 & 0.99999 \\
\hline & 148 & 8.83 & 33.89 & 0.0553 & 0.00067 & 0.31 & 0.99999 \\
\hline \multirow{3}{*}{ PMMA } & 60 & 3.33 & 452.45 & 0.6740 & 0.2028 & 0.81 & 0.99991 \\
\hline & 90 & 3.31 & 440.95 & 0.6569 & 0.2066 & 0.91 & 0.99988 \\
\hline & 120 & 3.44 & 423.15 & 0.6304 & 0.1804 & 1.00 & 0.99985 \\
\hline \multirow{3}{*}{ PC } & 60 & 3.97 & 359.15 & 3.820 & 0.0954 & 2.44 & 0.99906 \\
\hline & 90 & 3.71 & 374.00 & 3.98 & 0.1225 & 1.99 & 0.99941 \\
\hline & 120 & 3.53 & 384.53 & 4.090 & 0.1466 & 1.73 & 0.99954 \\
\hline \multirow{3}{*}{ PP } & 90 & 7.20 & 86.30 & 0.0813 & 0.0029 & 1.04 & 0.99986 \\
\hline & 107 & 7.14 & 84.30 & 0.0794 & 0.0031 & 0.82 & 0.99991 \\
\hline & 120 & 7.09 & 83.91 & 0.0790 & 0.0033 & 0.73 & 0.99993 \\
\hline \multirow{4}{*}{ PS } & 30 & 3.74 & 313.11 & 0.1990 & 0.1060 & 1.42 & 0.99970 \\
\hline & 60 & 3.71 & 303.36 & 0.1928 & 0.1091 & 1.11 & 0.99981 \\
\hline & 90 & 3.70 & 297.94 & 0.1893 & 0.1105 & 1.25 & 0.99977 \\
\hline & 120 & 3.63 & 296.65 & 0.1886 & 0.1178 & 1.48 & 0.99959 \\
\hline
\end{tabular}

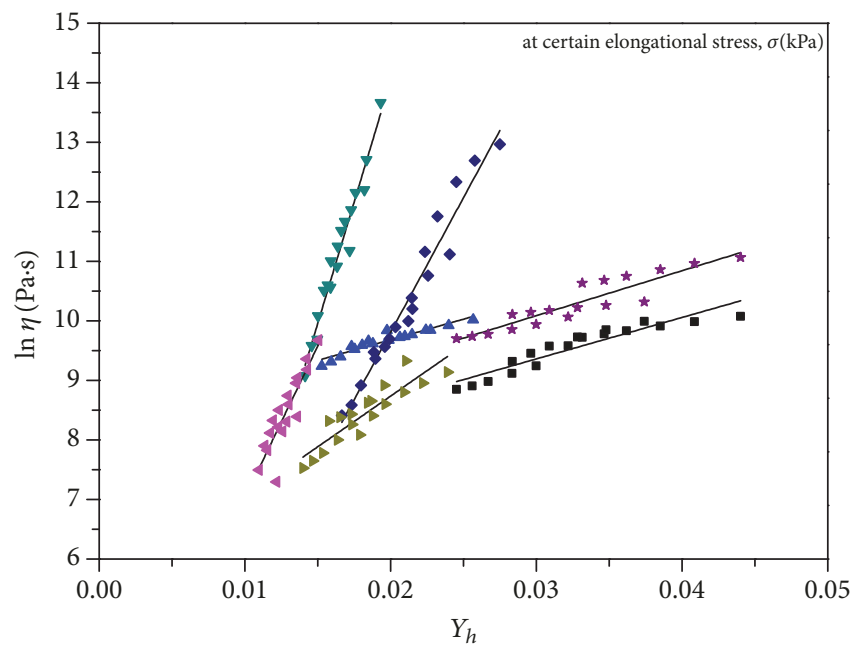

- LDPE 60

* LDPE 65

$\triangle$ HDPE

$\checkmark$ PMMA

$\triangleleft \mathrm{PC}$

- PP

- PS

FIGURE 6: The logarithm of viscosity at certain elongational stress (the elongational stresses used in the figure indicated with italic font in Table 4) versus $Y_{h}$. The solid line is the best fit line through each data set by (7) and (8) with the parameters tabulated in Table 4. 
TABLE 4: Values of parameters of (7) evaluated for $\eta_{E}$ at different elongational stresses.

\begin{tabular}{|c|c|c|c|c|c|c|c|}
\hline Polymer & $\sigma_{c}(\mathrm{kPa})$ & $\ln \eta_{\sigma}^{*}$ & $\alpha_{\sigma}$ & $a_{\sigma}^{\prime} \times\left(10^{4}\right)$ & $\kappa$ & $\Delta \eta_{E}(\%)$ & $R^{2}$ \\
\hline \multirow{4}{*}{ LDPE60 } & 500 & 7.27 & 69.62 & 0.136 & 0.0027 & 1.31 & 0.99980 \\
\hline & 800 & 6.74 & 83.59 & 0.163 & 0.0046 & 1.56 & 0.99969 \\
\hline & 1100 & 6.57 & 87.59 & 0.1711 & 0.0055 & 1.57 & 0.99966 \\
\hline & 1400 & 6.54 & 87.68 & 0.1713 & 0.0057 & 1.45 & 0.99971 \\
\hline \multirow{4}{*}{ LDPE65 } & 350 & 8.41 & 58.14 & 0.115 & 0.00086 & 2.85 & 0.99846 \\
\hline & 500 & 8.15 & 64.45 & 0.128 & 0.0011 & 1.54 & 0.99963 \\
\hline & 800 & 7.84 & 74.56 & 0.148 & 0.0015 & 1.31 & 0.99975 \\
\hline & 1100 & 7.77 & 77.07 & 0.152 & 0.0016 & 1.43 & 0.99968 \\
\hline \multirow{3}{*}{ LLDPE } & 800 & 8.15 & 70.54 & 0.189 & 0.0011 & 4.56 & 0.99725 \\
\hline & 1100 & 7.46 & 84.85 & 0.23 & 0.0022 & 3.57 & 0.99797 \\
\hline & 1400 & 6.24 & 112.06 & 0.30 & 0.0076 & 4.97 & 0.99642 \\
\hline \multirow{6}{*}{ HDPE } & 500 & 9.89 & 33.07 & 0.0540 & 0.00023 & 1.15 & 0.99980 \\
\hline & 800 & 9.25 & 53.52 & 0.0874 & 0.00044 & 0.59 & 0.99992 \\
\hline & 1100 & 8.83 & 62.72 & 0.102 & 0.00066 & 0.70 & 0.99992 \\
\hline & 1400 & 8.53 & 67.19 & 0.110 & 0.00089 & 0.61 & 0.99994 \\
\hline & 1700 & 8.30 & 69.22 & 0.113 & 0.0011 & 0.47 & 0.99996 \\
\hline & 2000 & 8.11 & 69.84 & 0.12 & 0.0014 & 0.59 & 0.99995 \\
\hline \multirow{3}{*}{ PMMA } & 1100 & -4.16 & 949.92 & 1.42 & 362.15 & 2.73 & 0.99840 \\
\hline & 1400 & -2.89 & 861.9 & 1.28 & 101.70 & 1.82 & 0.99934 \\
\hline & 1700 & -2.28 & 815.85 & 1.22 & 54.98 & 1.64 & 0.99960 \\
\hline \multirow{6}{*}{ PC } & 500 & 1.93 & 510.04 & 5.42 & 0.729 & 2.40 & 0.99895 \\
\hline & 800 & 1.49 & 536.48 & 5.71 & 1.136 & 2.78 & 0.99859 \\
\hline & 1100 & 1.18 & 554.40 & 5.90 & 1.535 & 3.32 & 0.99810 \\
\hline & 1400 & 0.96 & 567.97 & 6.04 & 1.927 & 3.76 & 0.99759 \\
\hline & 1700 & 0.77 & 578.90 & 6.16 & 2.314 & 4.30 & 0.99708 \\
\hline & 2000 & 0.62 & 588.04 & 6.25 & 2.698 & 4.76 & 0.99659 \\
\hline \multirow{3}{*}{$\mathrm{PP}$} & 350 & 7.99 & 55.21 & 0.052 & 0.0013 & 2.34 & 0.99885 \\
\hline & 500 & 6.37 & 137.53 & 0.13 & 0.0066 & 2.44 & 0.99915 \\
\hline & 800 & 5.33 & 170.44 & 0.161 & 0.0188 & 2.18 & 0.99940 \\
\hline \multirow{4}{*}{ PS } & 500 & 2.93 & 354.33 & 0.226 & 0.237 & 2.70 & 0.99849 \\
\hline & 800 & 1.37 & 427.57 & 0.27 & 1.128 & 2.34 & 0.99896 \\
\hline & 1100 & 0.89 & 448.55 & 0.285 & 1.82 & 1.98 & 0.99925 \\
\hline & 1400 & 0.77 & 452.19 & 0.287 & 2.068 & 1.93 & 0.99935 \\
\hline
\end{tabular}

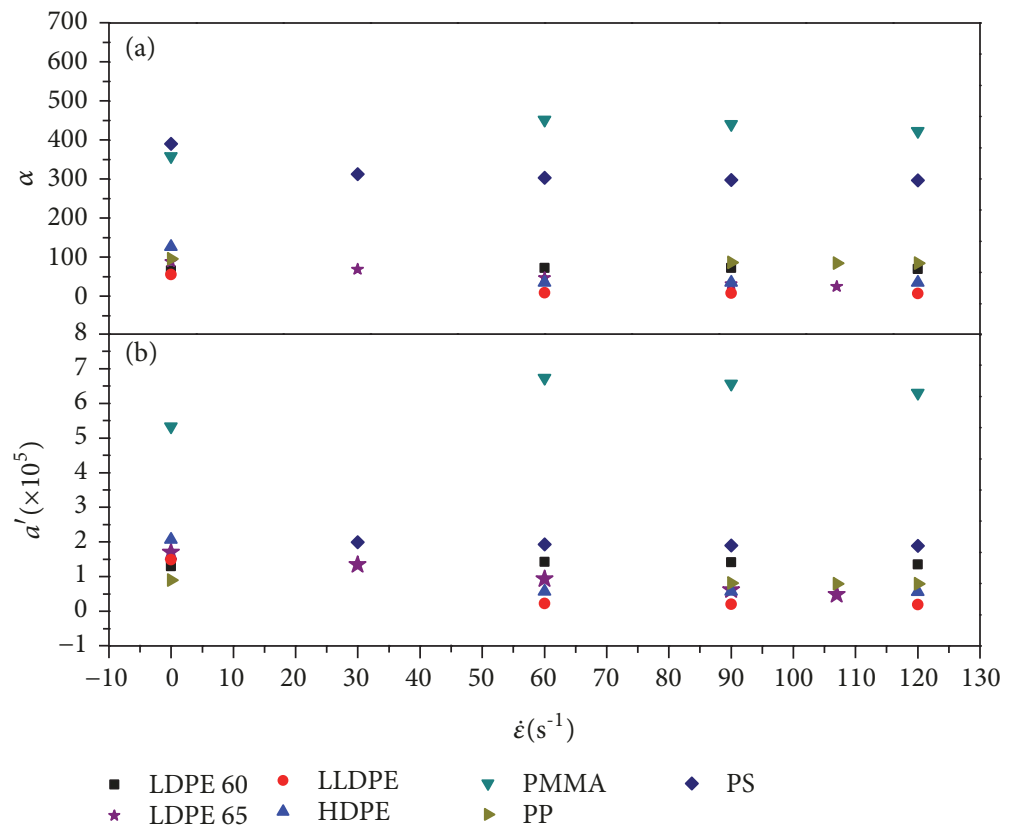

FIGURE 7: The elongational rate dependency of computed parameters $\alpha$ and $a^{\prime}$ calculated by (7) and (8). 


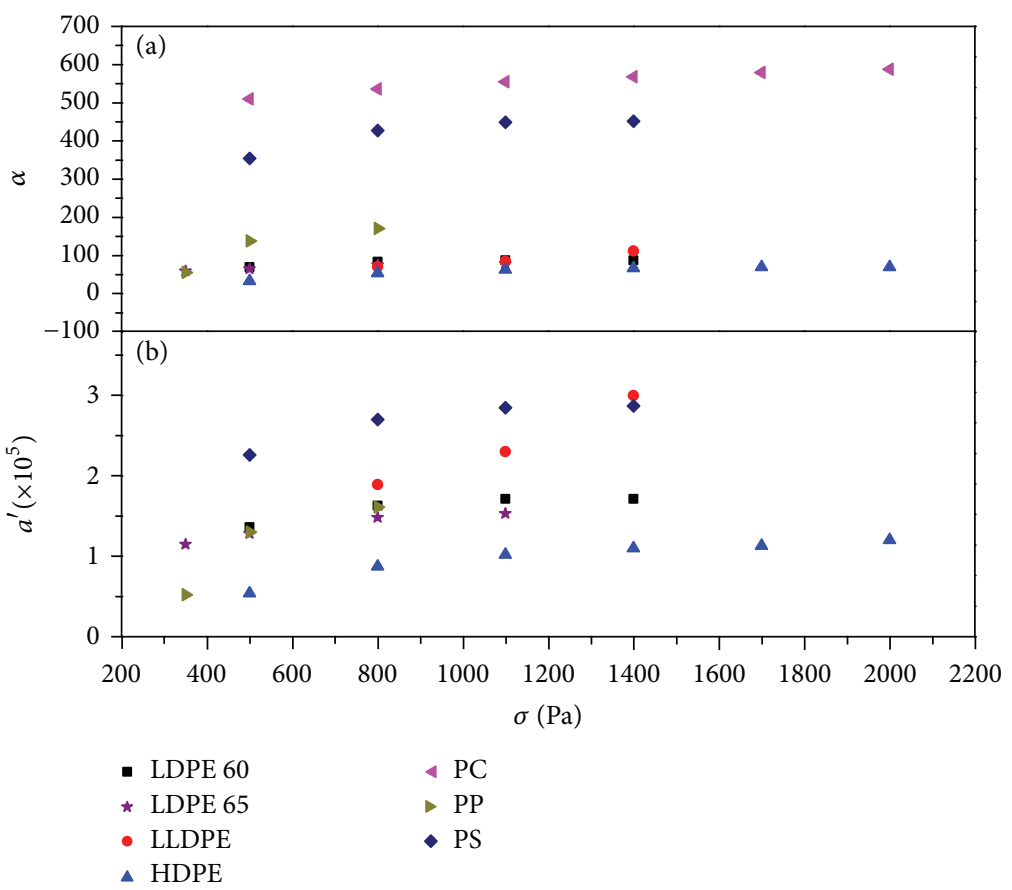

FIGURE 8: The elongational stress dependency of computed parameters $\alpha$ and $a^{\prime}$ calculated by (7) and (8).

constant elongational rates of $0,60,90$, and $120 \mathrm{~s}^{-1}$, the slopes of the lines for HDPE are 126.93, 35.23, 35.15, and 34.58, respectively. As can be seen the slopes of the lines are inversely correlated to elongational rates. This can be explained by the fact that, with the increasing $\dot{\varepsilon}$, the flowability increases, so $\alpha_{\dot{\varepsilon}}$ decreases. The same pattern is observed for the other polymer types except PC $\left(\alpha_{\dot{\varepsilon}}\right.$ and $a_{\dot{\varepsilon}}^{\prime}$ directly related to elongational rate) in Figure $7(\mathrm{a})$. A quite slight decrement is observed since the values of the strain rates and strain rate ranges are smaller in the elongational flow (3-405 s $\left.\mathrm{s}^{-1}\right)$ than in the shear flow $\left(0.1-8500 \mathrm{~s}^{-1}\right)$ [6]. The activation energy coefficient, $a_{\dot{\varepsilon}}^{\prime}$, is calculated for each polymer given in Table 3 and plotted in Figure 7(b). The behavior of $a_{\dot{\varepsilon}}^{\prime}$ is similar to $\alpha_{\dot{\varepsilon}}$. Figure 8 shows the change of $\alpha_{\sigma}$ and $a_{\sigma}^{\prime}$ parameters with elongational stress for the polymers given in Table 4 . These parameters slightly increase with elongational stress. However, in our previous work where the stress values are much lower than the elongational stress values as expected, $\alpha_{\sigma}$ and $a_{\sigma}^{\prime}$ stay almost constant with shear stress. Figure 8 is drawn with the exception of PMMA since $\alpha_{\sigma}$ and $a_{\sigma}^{\prime}$ inversely related to elongational stress. PC is also excluded in Figure 8(b) because of its largest values. In comparison, PMMA, PC, and PS have higher $\alpha$ and $a^{\prime}$ values than polyolefins. As noticed above, this can be arising from the fact that, bulky pendant groups attached to the backbone of these polymers make them more elongated and increase the intermolecular friction (viscosity). As the viscosity increases, the activation energy also increases. Consequently, the more activation energy requires the higher values of $\alpha$ and $a^{\prime}$.

Further, the transmission coefficient, $\kappa$, in (8) is also calculated and plotted in Figures $9(\mathrm{a})$ and 10 with respect to the elongational rate and the elongational stress, respectively. It has been found that the transmission coefficient, $\kappa$, increases linearly along with the increasing elongational rate and elongational stress. In Figure 9(a), there is a slight increment because of the low and small range of elongational rates. This rise enables the molecules to jump into the holes much more easily. On the other hand, the tendency of the molecules to the flowability decreases the intercepts, $\ln \eta_{E}^{*}$, at the viscosity axis as shown in Figures 9(b) and 11. $\ln \eta_{E}^{*}$ represents the extrapolated viscosity values when the system dominates sufficiently large hole fraction expectedly at high temperature and low pressure in (7). In other words, the thermooccupancy function, $Y_{h}$, goes to zero. This contrary behavior of $\kappa$ to $\ln \eta_{E}^{*}$ is obvious from Tables 3 and 4. Graphical results of this observation are depicted in Figures 9-11. Increasing the constant elongational rate given in Table 3 and the constant elongational stress given in Table 4, the intercepts for HDPE decrease steadily as 9.26, 8.98, 8.83 $\mathrm{Pa} \cdot \mathrm{s}$ and 9.25, 8.53, 8.11 $\mathrm{Pa} \cdot \mathrm{s}$, respectively. The decrements for the elongational rate and the elongational stress are also observed for the other polymers shown in Figures 9(b) and 11. LDPE 65 in Figure 9 and PMMA in Figure 11 are excluded, because of the increments with elongational rate and stress, respectively. From Figures 9-11 we understand that the calculated values of $\kappa$ of the constant elongational rate and stress of PC, PS, and PMMA are larger than of the polyolefins while they have smaller values of $\ln \eta_{E}^{*}$. This can be interpreted, such that the polymers, PMMA, PC, and PS, have bulky and larger pendant groups as allyl, methyl, and phenyl. Since the polymers with the side groups are very sensitive to produce more free volume and less viscous behavior as going to higher temperature or lower pressure, they take the higher values of $\kappa$ and the 


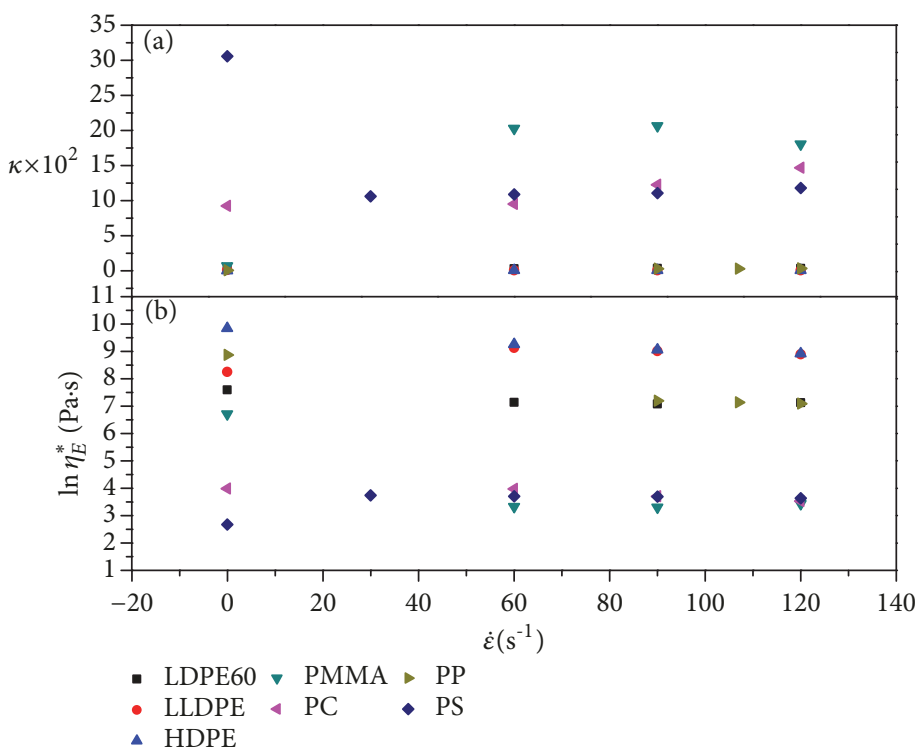

FIGURE 9: The elongational rate dependency of computed $\kappa$ and $\ln \eta_{E}^{*}$ calculated by (7) and (8).
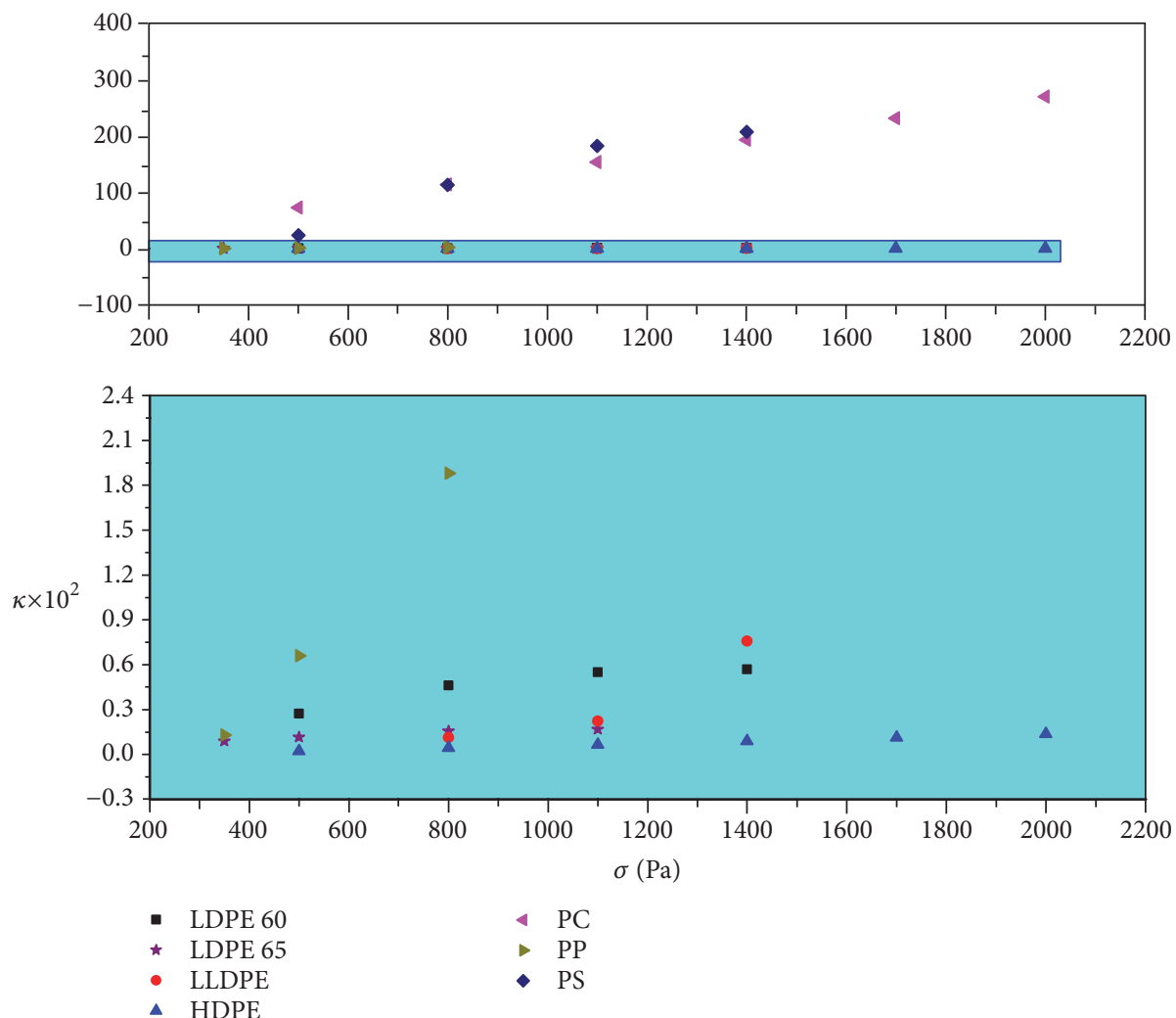

FIGURE 10: The elongational stress dependency of computed $\kappa$ calculated by (7) and (8).

smaller values of $\ln \eta_{E}^{*}$. The calculated values of $\ln \eta_{E}^{*}$ of the constant elongational stress order of the materials are as follows: HDPE $>$ LLDPE $>$ LDPE $>$ PP $>$ PC $>$ PS $>$ PMMA. The transmission coefficient, $\kappa$, order for the constant elongational stress is vice versa. This result is the same for the shear viscosity we obtained before (see [6]).

\section{Conclusions}

The Yahsi-Dinc-Tav (YDT) non-Newtonian viscosity equation and the Cross-like model were employed to describe the correlation among the elongational viscosity and elongational rate as well as the rheological parameters of polymer 


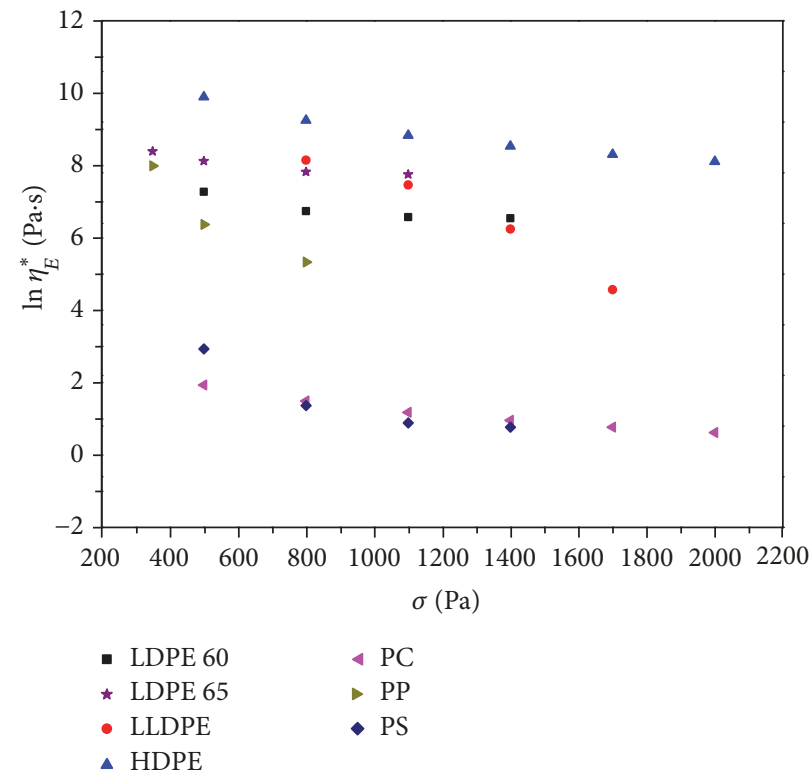

FIGURE 11: The elongational stress dependency of computed $\ln \eta_{E}^{*}$ calculated by (7) and (8).

materials. The results from the YDT model showed that the estimations were in good agreement with the experimental data both at ambient and at high pressures. In contrast to the findings for shear rate in our previous work (where the Cross model better fitted the data at ambient pressure), in this paper, the YDT model provides $0.5 \%$ less deviation than the Cross model at ambient pressure. The YDT equation gives better results than the Cross-like model not only at ambient pressure but also at elevated pressure. The mean percentage error decreases almost $0.8 \%$ at higher pressure by the use of the YDT model. Analyses also show that good estimation is observed for the polymers with high elongational rates (up to about $405 \mathrm{~s}^{-1}$ and $308 \mathrm{~s}^{-1}$ for PC and LLDPE, respectively). Moreover, the viscosity parameters determined from shear (from our earlier paper) and elongational flow maintained the similar temperature and pressure dependence. In brief, with this model, correct nonlinear behavior in both shear and elongation was achieved for the linear, branched, and large group attached polymers.

Regarding the dependence of viscosities on thermooccupancy function $\left(Y_{h}\right)$, the YDT-derived linear equations, at zero level of elongational and constant elongational rates as well as at constant elongational stress, provide a good description of dependency of hole fraction on viscosity. It was found that, for individual polymers, a linear relationship exists between the logarithm of zero elongational, constant elongational rate, and constant elongational stress viscosities (at several temperatures and pressures) and $Y_{h}$. Similar to the results we obtained in our earlier study for the shear viscosity, we show that an increase in thermooccupancy function results in higher viscosity values at various elongational rate and elongational stress conditions, since decrease in hole fraction causes stronger interactions between molecules. Polymers with the simpler structures, (HDPE, LLDPE, LDPE, and PP) appear to have the lower thermooccupancy function dependence. Larger pendant groups linked to the backbone chains of the remaining polymers (PC, PS, and PMMA) result in a higher dependency on temperature- and pressuredependent thermooccupancy function.

Further, the accessibility of rheological data over a broad range of pressures and temperatures and the hole fraction computed from the SS theory allow the viscosity parameters such as activation energy and transmission coefficients to be computed from such linear formulations. The changes in these viscosity parameters are discussed in terms of constant elongation rate and constant elongational stress. Estimates show that activation energy coefficient is negatively correlated with elongational rate. With regard to the elongational stress, it has a direct relationship contrary to what we have found for shear viscosity. Confirming the results, we have had for shear flow, transmission coefficient is positively correlated with constant elongational rate and constant elongational stress, while the viscosity-intercept has an inverse relationship.

In summary, the model predictions for the nonlinear behavior in both elongation and shear viscosities in this and our preceding work have been revealed to be in very good agreement with the reported experimental data. This viscosity equation in experimental studies is reliable and functional with only a fitting parameter as well as convenient not only for linear polymers, but also for the polymers with long chain branching and large attached groups. An intriguing forthcoming study may be on predicting other physical properties of different classes of neat polymers and their compositions with a similar methodology followed in the current work.

\section{Nomenclature} $a^{\prime}:$ Proportionality constant of activation
energy

$3 c$ : Total external degrees of freedom 


\begin{tabular}{|c|c|}
\hline$E_{a}:$ & Activation energy $(\mathrm{J})$ \\
\hline$h_{p}:$ & Planck constant $\left(6.626 \times 10^{-34} \mathrm{~J} . \mathrm{s}\right)$ \\
\hline$h:$ & Hole fraction \\
\hline$k:$ & Boltzmann's constant $\left(1.38 \times 10^{-23} \mathrm{~J} / \mathrm{K}\right)$ \\
\hline$m_{0}:$ & Segmental molar mass of molecules (kg) \\
\hline$N_{A}:$ & Avogadro's number \\
\hline$\widetilde{P}, \widetilde{V}, \widetilde{T}:$ & $\begin{array}{l}\text { Reduced pressure, volume, and } \\
\text { temperature }\end{array}$ \\
\hline$P^{*}, V^{*}, T^{*}:$ & $\begin{array}{l}\text { Characteristic pressure, volume, and } \\
\text { temperature }\end{array}$ \\
\hline Q: & $\begin{array}{l}\text { Hole fraction and reduced volume } \\
\text { dependent quantity in (3) and (4) }\end{array}$ \\
\hline$q:$ & $\begin{array}{l}\text { Nonlinearity parameter in the power of } \\
\text { elongational rate }\end{array}$ \\
\hline$q_{z}:$ & $\begin{array}{l}\text { Number of interchain nearest neighbor } \\
\text { pairs in a lattice of coordination number }\end{array}$ \\
\hline$R:$ & Gas constant $(8.314 \mathrm{~J} / \mathrm{mol} \mathrm{K})$ \\
\hline$s:$ & Number of segments of molecules \\
\hline$T:$ & Temperature $\left({ }^{\circ} \mathrm{C}\right.$ or $\left.\mathrm{K}\right)$ \\
\hline$v:$ & Molar segmental volume \\
\hline$v^{*}$ & Characteristic molar volume of a segment \\
\hline$y:$ & Occupied site fraction \\
\hline$Y_{h}$ & “Thermooccupancy function", in (7) \\
\hline & Coordination number. \\
\hline
\end{tabular}

\section{Greek Letters}

$\alpha$ : Slope of (7)

$\dot{\varepsilon}$ : Elongational rate $\left(\mathrm{s}^{-1}\right)$

$\dot{\varepsilon}_{c}$ : Constant elongational rate

$\dot{\varepsilon}_{0}$ : Zero level of elongational rate

$\begin{array}{ll}\Delta \eta_{E}(\%): & \text { Average percentage error in viscosity } \\ \varepsilon^{*}: & \begin{array}{l}\text { Attractive interaction parameter of a } \\ \text { segment of the potential minimum }\end{array} \\ \eta_{E}^{*}: & \begin{array}{l}\text { Intercept of }(7) \text { and }(8) \\ \eta_{0}, \eta_{\dot{\varepsilon}}:\end{array} \\ \theta_{i}: & \begin{array}{l}\text { Zero level of elongational viscosity } \\ \text { constant elongational rate viscosity }(\mathrm{Pa} \cdot \mathrm{s})\end{array} \\ & \begin{array}{l}\text { The angle between the deformation stress } \\ \text { and the displacement vector of } i \text { th }\end{array} \\ \kappa: & \begin{array}{l}\text { contiguity of a segment } \\ \text { Transmission coefficient }\end{array} \\ & \begin{array}{l}\text { Interaction potential energy between a } \\ \text { pair of segments }(k \cdot \mathrm{K})\end{array} \\ \sigma: & \begin{array}{l}\text { Elongational stress }\left(\mathrm{N} / \mathrm{m}^{2}\right)^{2} \\ \tau:\end{array}\end{array}$

\section{Data Availability}

The non-Newtonian viscosity and the PVT data supporting this theoretical analysis are from previously reported studies and datasets which were published by the one of the authors Sedlacek et al. The data have been cited in the text as $[4,11]$ for the elongational non-Newtonian viscosity and the PVT data, respectively. The processed data are available from the corresponding author upon request.

\section{Disclosure}

This research has not been financially funded by any special and governmental project and support. And also this work has not performed as part of the employment of the authors.

\section{Conflicts of Interest}

The authors declare that there are no conflicts of interest regarding the publication of this paper.

\section{References}

[1] J. Meissner, "Polymer melt elongation-methods, results, and recent developments," Polymer Engineering \& Science, vol. 27, no. 5, pp. 537-546, 1987.

[2] H. M. Laun and H. Munstedt, "Comparison of the elongational behaviour of a polyethylene melt at constant stress and constant strain rate," Rheologica Acta, vol. 15, no. 10, pp. 517-524, 1976.

[3] M. Gupta, "Estimation of elongational viscosity of polymers from entrance loss data using individual parameter optimization," Advances in Polymer Technology, vol. 21, no. 2, p. 98, 2002.

[4] T. Sedlacek, M. Zatloukal, P. Filip, A. Boldizar, and P. Saha, "On the effect of pressure on the shear and elongational viscosities of polymer melts," Polymer Engineering \& Science, vol. 44, p. 1328, 2004.

[5] J. M. Zatloukal, "Differential viscoelastic constitutive equations for polymer melts in steady shear and elongational flows," Journal of Non-Newtonian Fluid Mechanics, vol. 113, no. 2-3, pp. 209-227, 2003

[6] F. S. Dinc, T. Sedlacek, C. Tav, and U. Yahsi, "On the nonnewtonian viscous behavior of polymer melts in terms of temperature and pressure-dependent hole fraction," Journal of Applied Polymer Science, vol. 131, article no. 40540, no. 15, 2014.

[7] L. A. Utracki, "Pressure dependence of newtonian viscosity," Polymer Engineering \& Science, vol. 23, no. 8, pp. 446-451, 1983.

[8] L. A. Utracki, "A method of computation of the pressure effect on melt viscosity," Polymer Engineering \& Science, vol. 25, pp. 655-668, 1985.

[9] L. A. Utracki, "Temperature and pressure dependence of liquid viscosity," The Canadian Journal of Chemical Engineering, vol. 61, no. 5, pp. 753-758, 1983.

[10] L. A. Utracki and T. Sedlacek, "Free volume dependence of polymer viscosity," Rheologica Acta, vol. 46, pp. 479-494, 2007.

[11] T. Sedlacek, R. Cermak, B. Hausnerova, M. Zatloukal, A. Boldizar, and P. Saha, "On PVT and rheological measurements of polymer melts: Correction of the hole fraction-viscosity relationship," International Polymer Processing, vol. 20, no. 3, pp. 286-295, 2005.

[12] U. Yahsi and F. Sahin, "Linking the viscous and vacancy behavior of high molecular weight hydrocarbons," Rheologica Acta, vol. 43, no. 2, pp. 159-167, 2004.

[13] F. Sahin-Dinc, A. Sorrentino, C. Tav, and U. Yahsi, "The effect of hole fraction on viscosity in atactic and syndiotactic polystyrenes," International Journal of Thermophysics, vol. 36, no. 10-11, pp. 3239-3254, 2015.

[14] F. Sahin-Dinc, "Hole fraction dependence on linear viscosity of PS, PP and ABS," International Polymer Processing, vol. 30, no. 5 , p. 585, 2015. 
[15] J. D. van der Waals, Over de continuiteit van den gas-en vloeistoftoestand (on the continuity of the gas and liquid state). [Ph.D. Thesis], University of Leiden, Leiden, Netherlands, 1873.

[16] A. J. Batchinski, Untersuchungen über die innere Reibung der Flussigkeiten, vol. 84, p. 643, 1913.

[17] A. K. Doolittle, "Studies in newtonian flow. II. The dependence of the viscosity of liquids on free-space," Journal of Applied Physics, vol. 22, p. 1471, 1951.

[18] A. K. Doolittle and D. B. Doolittle, "Studies in Newtonian flow. V. Further verification of the free-space viscosity equation," Journal of Applied Physics, vol. 28, p. 901, 1957.

[19] R. Simha and T. Somcynsky, "On the statistical thermodynamics of spherical and chain molecule fluids," Macromolecules, vol. 2, no. 4, pp. 342-350, 1969.

[20] U. Yahsi, K. Ulutas, C. Tav, and D. Deger, "On the ionic conductivity of polymer electrolytes in terms of hole fraction," Polymer Physics, vol. 46, no. 20, p. 2249, 2008.

[21] H. M. Laun and H. Munstedt, "Elongational behaviour of a low density polyethylene melt," Rheologica Acta, vol. 17, no. 4, pp. 415-425, 1978. 


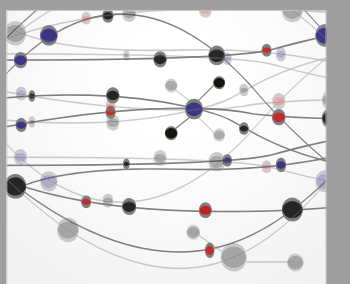

The Scientific World Journal
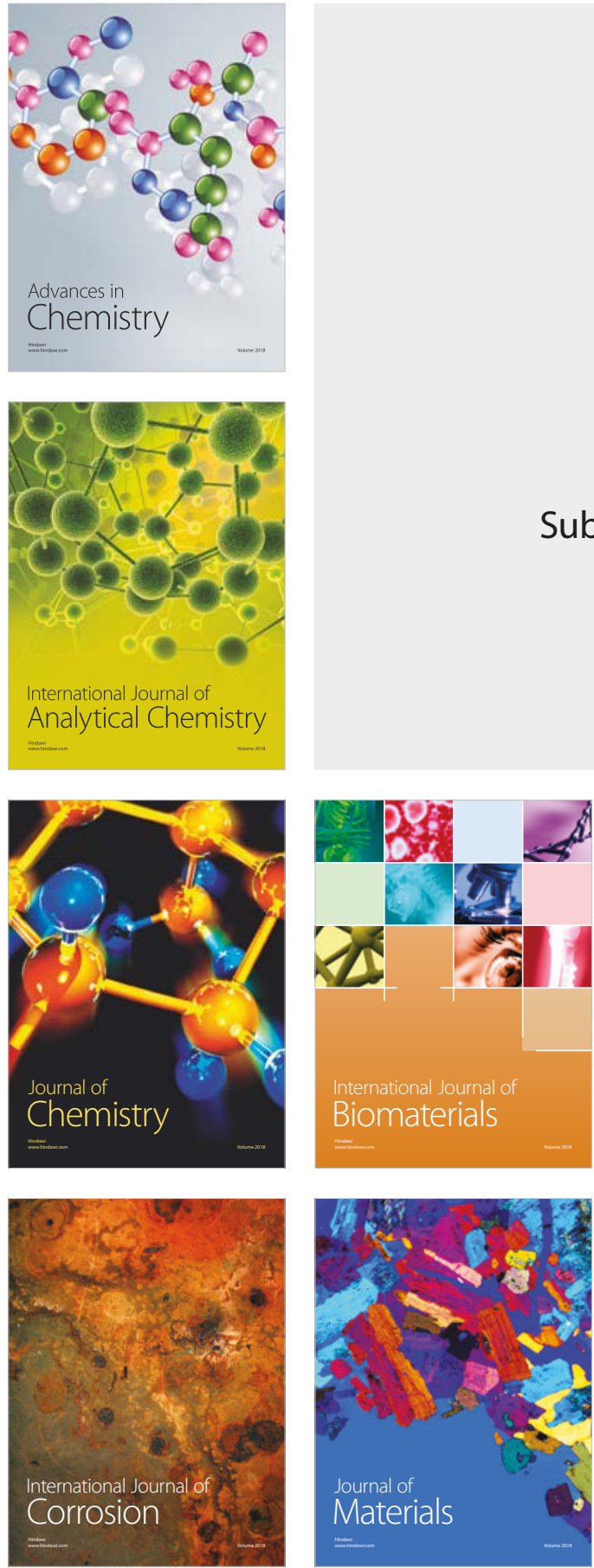

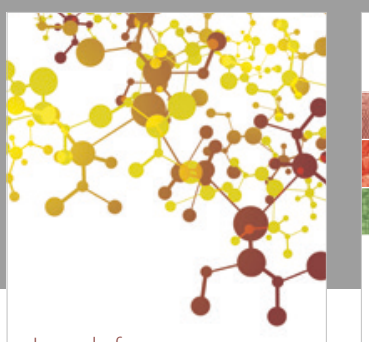

Journal of

Applied Chemistry
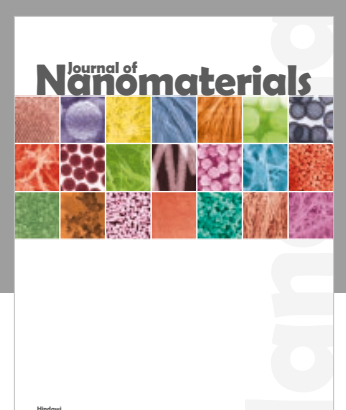

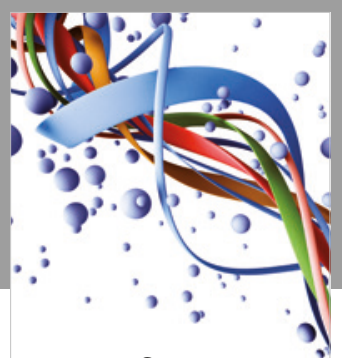

Scientifica

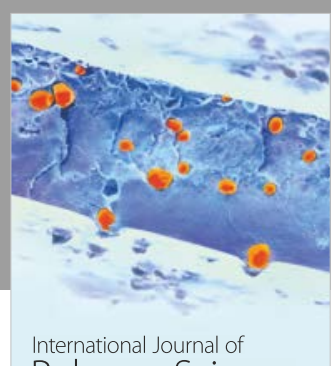

Polymer Science

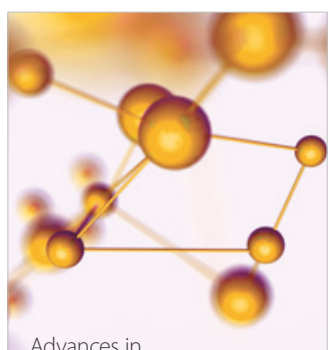

Physical Chemistry
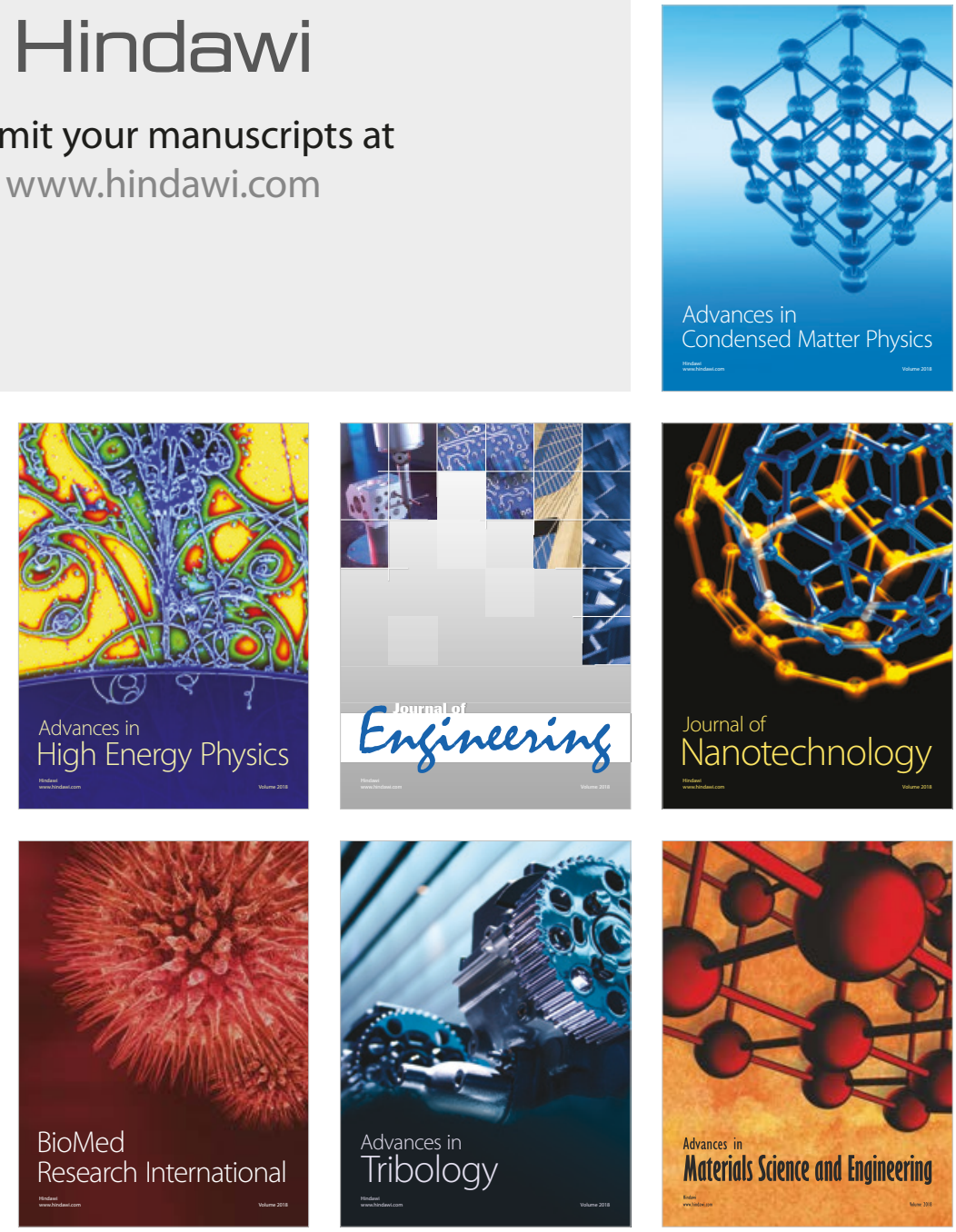